ISSN: 2053-230X

journals.iucr.org/f

\title{
Crystal structure of Arabidopsis thaliana neutral invertase 2
}

\section{Łukasz P. Tarkowski, Vicky G. Tsirkone, Evgenii M. Osipov, Steven Beelen, Willem Lammens, Rudy Vergauwen, Wim Van den Ende and Sergei V. Strelkov}

Acta Cryst. (2020). F76, 152-157

\begin{tabular}{|l} 
IUCr Journals \\
CRYSTALLOGRAPHY JOURNALS ONLINE \\
Copyright (C) International Union of Crystallography \\
Author(s) of this article may load this reprint on their own web site or institutional repository provided that \\
this cover page is retained. Republication of this article or its storage in electronic databases other than as \\
specified above is not permitted without prior permission in writing from the IUCr. \\
For further information see https://journals.iucr.org/services/authorrights.html
\end{tabular}


STRUCTURAL BIOLOGY COMMUNICATIONS

ISSN 2053-230X

Received 22 August 2019

Accepted 7 February 2020

Edited by N. Sträter, University of Leipzig, Germany

₹ Current address: Seed Metabolism and Stress Team, INRAE Angers, Institut de Recherche en Horticulture et Semences, Bâtiment A, 42 Rue Georges Morel, CS 60057, 49071 Beaucouzé CEDEX, France.

$\S$ These authors contributed equally to this work.

Keywords: GH100; neutral invertase; Arabidopsis thaliana; crystal structure.

PDB reference: neutral invertase 2 from Arabidopsis thaliana, $6 \mathrm{ttj}$

Supporting information: this article has supporting information at journals.iucr.org/f

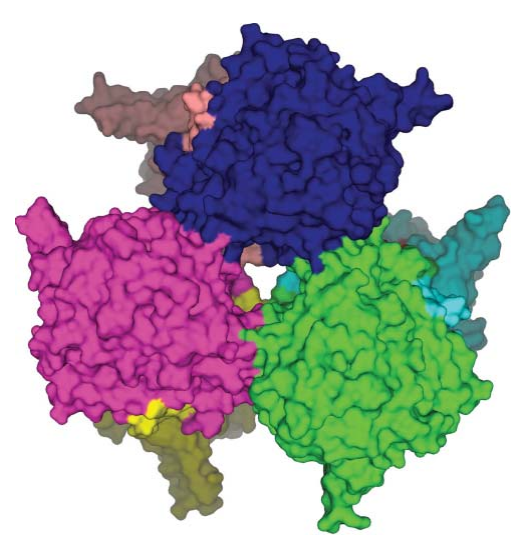

C 2020 International Union of Crystallography

\section{Crystal structure of Arabidopsis thaliana neutral invertase 2}

\author{
Łukasz P. Tarkowski, ${ }^{\mathrm{a}} £ \S$ Vicky G. Tsirkone, ${ }^{\mathrm{a}} \S$ Evgenii M. Osipov, ${ }^{\mathrm{a}} \S$ Steven Beelen, \\ Willem Lammens, ${ }^{a}$ Rudy Vergauwen, ${ }^{a}$ Wim Van den Ende ${ }^{a}$ and Sergei V. Strelkov ${ }^{b *}$
}

${ }^{a}$ Molecular Plant Biology Laboratory, Department of Biology, KU Leuven, Kasteelpark Arenberg 31, 3000 Leuven, Belgium, and ${ }^{\mathbf{b}}$ Laboratory for Biocrystallography, Department of Pharmaceutical and Pharmacological Sciences, KU Leuven, Herestraat 49, O\&N II, bus 822, 3000 Leuven, Belgium. *Correspondence e-mail: sergei.strelkov@kuleuven.be

The metabolism of sucrose is of crucial importance for life on Earth. In plants, enzymes called invertases split sucrose into glucose and fructose, contributing to the regulation of metabolic fluxes. Invertases differ in their localization and $\mathrm{pH}$ optimum. Acidic invertases present in plant cell walls and vacuoles belong to glycoside hydrolase family 32 (GH32) and have an all- $\beta$ structure. In contrast, neutral invertases are located in the cytosol and organelles such as chloroplasts and mitochondria. These poorly understood enzymes are classified into a separate GH100 family. Recent crystal structures of the closely related neutral invertases InvA and InvB from the cyanobacterium Anabaena revealed a predominantly $\alpha$-helical fold with unique features compared with other sucrosemetabolizing enzymes. Here, a neutral invertase (AtNIN2) from the model plant Arabidopsis thaliana was heterologously expressed, purified and crystallized. As a result, the first neutral invertase structure from a higher plant has been obtained at $3.4 \AA$ resolution. The hexameric AtNIN2 structure is highly similar to that of InvA, pointing to high evolutionary conservation of neutral invertases.

\section{Introduction}

Plants are autotrophic organisms that are able to sustain themselves by fixating atmospheric carbon through photosynthesis. This process generates sucrose (Suc), which is used as a primary source of organic carbon in plant metabolism (Winter \& Huber, 2000). Besides its metabolic functions, Suc acts as a signalling molecule, with roles in developmental programs and stress responses (Van den Ende \& El-Esawe, 2014; Ruan, 2014). In plants, Suc can be catabolized by the action of sucrose synthase (SuSy; EC 2.4.1.13) or invertases (Winter \& Huber, 2000). Whereas SuSy catalyzes a reversible reaction yielding nucleotide diphosphate glucose and fructose (Fru; Stein \& Granot, 2019), invertases split Suc irreversibly into glucose (Glc) and Fru without the use of cofactors (Sturm \& Tang, 1999; Koch, 2004). The contribution of invertases to Suc metabolism is crucial in sink initiation and early organ development, while the contribution of SuSy becomes prevalent during storage and maturation phases, playing a role in starch accumulation and the synthesis of complex polysaccharides (Koch, 2004).

Invertases are classified into two subgroups based on their $\mathrm{pH}$ optimum: acidic invertases and neutral/alkaline invertases 
(NIs) (Sturm, 1999). Acidic invertases are divided by their subcellular localization into cell-wall invertases and vacuolar invertases. NIs can be found in the cytosol and in organelles such as chloroplasts, the nucleus and mitochondria (Shen et al., 2018). In the past, research has mostly focused on acidic invertases because of their importance in source-sink balance and fruit development, and their contribution to biotic and abiotic stress responses (Roitsch et al., 2003; Tauzin \& Giardina, 2014; Palmer et al., 2015). In contrast, NIs have gained little attention until recently (Vargas \& Salerno, 2010), but new evidence has highlighted their importance in processes such as cellulose synthesis, plastidial retrograde signalling and light/dark signalling (Gao et al., 2014; Maruta et al., 2015; Barnes \& Anderson, 2018).

Acidic invertases are expressed from genes containing six to eight exons and reveal highly conserved structural features and catalytic mechanisms. These enzymes hydrolyze the glycosidic bond of Suc, which is their common substrate. However, cases of substrate promiscuity have been documented towards 1 -kestose ( $\mathrm{F}^{\mathrm{F}}-\beta$-D-fructosylsucrose $)$ and raffinose $\left(6^{\mathrm{G}}-\alpha\right.$-D-galactosylsucrose) (Trollope et al., 2015). Acidic invertases include an $\mathrm{N}$-terminal $\beta$-propeller domain, which contains the active site, and a C-terminal $\beta$-sandwich domain (Alberto et al., 2004). Enzymes with such characteristics are classified into glycoside hydrolase family 32 (GH32), which also contains all enzymes that are capable of metabolizing fructans (Altenbach \& Ritsema, 2007). These enzymes are structurally very similar, but are functionally different owing to only a few mutations in their active sites (Van den Ende et al., 2009).

In contrast, NIs have multiple distinct features when compared with acidic invertases, with a stringent substrate specificity for Suc (Qi et al., 2007; Xie et al., 2016), and are classified as the only member of the GH100 family. NIs are thought to be derived from cyanobacteria, which lack acidic invertases (Vargas \& Salerno, 2010). Since open-ocean algal strains also lack SuSy homologs, these authors hypothesized that NI-like enzymes might represent the origin of sucrose metabolism (Vargas \& Salerno, 2010), thus highlighting their evolutionary importance for the development of life. Recently, the crystal structures of two closely related NIs from the cyanobacterium Anabaena sp. PCC 7120 (named InvA and InvB) were determined (Xie et al., 2016, 2018). These first structures of GH100 enzymes confirmed the unique properties of NIs compared with acidic invertases, such as a predominantly $\alpha$-helical structure and a specific catalytic mechanism that is selective for Suc. Both proteins have an $(\alpha / \alpha)_{6}$-barrel fold composed of 12 helices, while InvB reveals a much higher activity compared with InvA (Xie et al., 2016, 2018). Here, we aimed to heterologously express, purify and crystallize an NI from the model plant Arabidopsis thaliana (AtNIN2; UniProt Q9LQF2) in order to obtain the first atomic structure of a higher plant NI. The crystal structure showed a strong similarity to those of the NIs from Anabaena, suggesting an evolutionarily conserved structure and catalytic mechanism. These data corroborate the hypothesis that plant NIs are derived from cyanobacteria.

\section{Materials and methods}

\subsection{Reagents}

Cloning enzymes were purchased from New England Biolabs (NEB; Ipswich, Massachusetts, USA) unless otherwise stated. All reagents for growth media were purchased from Thermo Fisher Scientific (Pittsburgh, Pennsylvania, USA). All other chemicals were purchased from Sigma-Aldrich (St Louis, Missouri, USA) unless otherwise stated. PCR primers were purchased from Integrated DNA Technologies (Belgium).

\subsection{Cloning, expression and purification of AtNIN2}

The AtNIN2 gene (AT1G35580, 552 residues) was amplified from the genomic DNA of $A$. thaliana and inserted into a modified pETSUL vector which coded for an $\mathrm{N}$-terminal $\mathrm{His}_{6}$ tag followed by the small ubiquitin-like modifier (SUMO) domain. The ligation-independent cloning procedure was performed as described in Weeks et al. (2007) using the following primers: ACCGCGAACAGATTGGTGGCATGG AAGGTGTTGGACTAAGAGCTG (forward) and CTTCT CGAGGAGAGTTTAGATTAGAGTTGTGGCCAAGACG CAG (reverse). The construct was overexpressed in Escherichia coli strain $\mathrm{DH} \alpha$ (Merck, Darmstad, Germany). Bacteria were grown in 51 culture medium $(10 \mathrm{~g}$ tryptone, $5 \mathrm{~g}$ yeast extract, $7.098 \mathrm{~g} \mathrm{NaH}_{2} \mathrm{PO}_{4}, 6.804 \mathrm{~g} \mathrm{KH}_{2} \mathrm{PO}_{4}, 3.3 \mathrm{~g} \mathrm{Na}_{2} \mathrm{SO}_{4}$ and $0.493 \mathrm{~g} \mathrm{MgSO}_{4}$ per litre) containing $0.5 \mathrm{~g} \mathrm{l}^{-1}$ glucose and

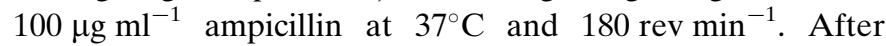
reaching an optical density of 0.6 at $600 \mathrm{~nm}, 2 \mathrm{~g} \mathrm{l}^{-1}$ lactose was added to induce protein expression. The cells were collected by centrifugation, resuspended in lysis buffer ( $8 \mathrm{~m} M$ imidazole, $1 \mathrm{~m} M \beta$-mercaptoethanol $\mathrm{pH} 7.5$ ), sonicated for $10 \mathrm{~min}$ and centrifuged at $20000 \mathrm{~g}$ for $30 \mathrm{~min}$. The supernatant containing the target protein as a $\mathrm{His}_{6}$-SUMO fusion was loaded onto a nickel-nitrilotriacetic acid column (GE Healthcare, Chicago, Illinois, USA). The target protein was eluted in a gradient to $300 \mathrm{~m} M$ imidazole in $5 \mathrm{ml}$ fractions. The fractions were tested for AtNIN2 activity by adding $10 \mu \mathrm{l}$ aliquots to $80 \mu \mathrm{l} 50 \mathrm{mM}$ TEA buffer $\mathrm{pH} 7.5$ and $10 \mu \mathrm{l} 0.5 \mathrm{M}$ Suc and incubating for $30 \mathrm{~min}$ at $30^{\circ} \mathrm{C}$. The activity was evaluated by quantifying the Fru peak upon elution using an HPLC ICS 3000 system (Thermo Fisher Scientific, Pittsburgh, Pennsylvania, USA). Fractions showing activity were tested for purity by gel electrophoresis and combined. They were subsequently incubated overnight with SUMO hydrolase to remove the $\mathrm{His}_{6}-\mathrm{SUMO}$ tag and loaded again onto the nickel-nitrilotriacetic acid column to capture the tag and uncleaved fusion protein. The cleaved protein was loaded onto a Superdex 200 column (Pharmacia Biotech, Piscataway, New Jersey, USA) and eluted in $1 \mathrm{ml}$ fractions at $2 \mathrm{ml} \mathrm{min}^{-1}$. Fractions with AtNIN2 activity were pooled together and concentrated to $\sim 10 \mathrm{mg} \mathrm{ml}^{-1}$ by ultrafiltration in Vivaspin tubes with $10 \mathrm{kDa}$ cutoff (Sartorius Stedim Biotech, Aubagne, France). The concentrated protein was evaluated on a gel for purity before crystallization trials. 
Table 1

Data-collection and refinement statistics.

Values in parentheses are for the highest resolution shell.

\begin{tabular}{ll}
\hline Data collection & \\
Space group & $P 2_{1}$ \\
Unit-cell parameters $\left(\AA{ }^{\circ}\right)$ & $a=107.5, b=186.7, c=152.2, \beta=104.9$ \\
Resolution range $(\AA)$ & $48.71-3.39(3.46-3.39)$ \\
Unique reflections & $79402(4000)$ \\
Mean $I / \sigma(I)$ & $4.8(1.2)$ \\
$R_{\text {merge }}$ & $0.415(1.698)$ \\
$R_{\text {merge }}$ in top intensity bin & 0.061 \\
Multiplicity & $3.7(3.3)$ \\
Completeness $(\%)$ & $99.0(87.6)$ \\
CC & $95.1(85.2)$ \\
Refinement & \\
$R_{\text {work }}$ & $0.253(0.41)$ \\
$R_{\text {free }}$ & $0.285(0.44)$ \\
Monomers per asymmetric unit & 12 \\
Total No. of non- $\mathrm{H}$ atoms & 42174 \\
Average $B$ factor $\left(\AA^{2}\right)$ & 60 \\
R.m.s. deviations & \\
Bond lengths $(\AA)$ & 0.005 \\
Bond angles $\left({ }^{\circ}\right)$ & 1.16 \\
Ramachandran plot statistics & \\
$\quad$ Favoured $(\%)$ & 94.56 \\
Outliers $(\%)$ & 0.44 \\
MolProbity score & 1.8
\end{tabular}

\subsection{Crystallization}

Initial screening for crystallization conditions was performed using the commercial kits Index (Hampton Research, Aliso Viejo, California, USA), Morpheus and Wizard Classic 1 \& 2 (Molecular Dimensions, Newmarket, England). Hanging drops consisting of $200 \mathrm{nl}$ protein sample at $10 \mathrm{mg} \mathrm{ml}^{-1}$ and $200 \mathrm{nl}$ precipitant solution were placed in 96-well plates using a Mosquito robot (TTP Labtech, Melbourn, England). Crystallization plates were incubated at either $4^{\circ} \mathrm{C}$ or $20^{\circ} \mathrm{C}$ and were monitored using a Rock Imager 1000 (Formulatrix, Bedford, Massachusetts, USA). Crystallization conditions were further optimized manually. The largest crystals $(500 \times 300 \times 300 \mu \mathrm{m})$ were obtained after $7 \mathrm{~d}$ using a reservoir solution consisting of $2.9 \mathrm{M} \mathrm{NaCl}, 9 \%$ polyethylene glycol 4000, $0.1 M$ bicine $\mathrm{pH} 9.0$.

\subsection{X-ray data collection}

Prior to data collection, crystals were soaked for $10 \mathrm{~s}$ in crystallization solution supplemented with $20 \%(v / v)$ glycerol and flash-cooled in liquid nitrogen. X-ray diffraction data were collected at $100 \mathrm{~K}$ on beamline ID30B at the ESRF, Grenoble, France using radiation of wavelength $0.98 \AA$ and an EIGER 9M detector (Dectris, Switzerland; McCarthy et al., 2018).

\subsection{Data processing and structural refinement}

The obtained diffraction data set was processed using $X D S$ (Kabsch, 2010). Scaling and merging were performed using AIMLESS (Evans \& Murshudov, 2013) from the CCP4 suite (Winn et al., 2011). The structure was solved by molecular replacement using the structure of InvA from Anabaena (PDB entry 5goo; 54\% identity; Xie et al., 2016) as a search model with MOLREP (Vagin \& Teplyakov, 2010). The structure was intially refined with REFMAC5 (Murshudov et al., 2011) using 'jelly-body' and local NCS restraints. The final structure was refined in phenix.refine (Liebschner et al., 2019) using geometry/ X-ray weight optimization, local NCS, Ramachandran-plot and reference-structure restraints. For the latter, PDB entry 5gop (Xie et al., 2016) was used as a reference. Manual correction of the model was performed in Coot (Emsley et al., 2010). Data-collection and refinement statistics are summarized in Table 1. The structure was validated using MolProbity (Williams et al., 2018) and deposited in the PDB with accession code $6 \mathrm{ttj}$. Images were prepared in PyMOL 2.0 (Schrödinger).

\section{Results and discussion \\ 3.1. Overall structure of AtNIN2}

AtNIN2 consists of 551 residues and has a molecular weight of $63 \mathrm{kDa}$ and a pI of 6.4. Optimization of the $\mathrm{NaCl}$ concentration, $\mathrm{pH}$ and additives (glycerol and $\beta$-mercaptoethanol) allowed us to obtain crystals of the full-length protein which diffracted to $3.4 \AA$ resolution. These crystals grew from $2.9 \mathrm{M}$ $\mathrm{NaCl}, 0.1 M$ bicine $\mathrm{pH} 9.0,9 \%$ PEG 4000. In comparison, fulllengh InvA crystals (Xie et al., 2016) were obtained using $27 \%$ PEG 6000, 0.1 $M$ bicine pH 9.0 and diffracted to $2.7 \AA$ resolution. Additionally, InvA was crystallized in a truncated form in the presence of Fru, revealing superior diffraction to $2.1 \AA$ resolution (Xie et al., 2016). While we could obtain a truncated AtNIN2 sample through limited proteolysis, this protein did not crystallize. Furthermore, addition of Fru to the crystallization condition did not improve the diffraction quality of the AtNIN2 crystals.

The asymmetric unit of the AtNiN2 crystal contains 12 monomers with a pairwise r.m.s.d. of less than $0.3 \AA$ (Fig. 1). 444 AtNIN2 residues per monomer were located in electrondensity maps. An extensive N-terminal region (residues Met1Pro81), the surface loop Asn112-Asn119 and the very C-terminal region (Glu534-Leu551) were not visible in any of the 12 monomers. The monomeric fold corresponds to an $(\alpha / \alpha)_{6}$-barrel, although the lengths and positions of the 12 concentrically arranged $\alpha$-helices are not entirely uniform. Of those, six helices form the inner barrel fold, while six additional helices are located on the outside, supplemented by surface loops including short $\beta$-hairpins (Fig. 2). Interestingly, the asymmetric unit reveals two identical hexamers with 32 point symmetry. Such a hexamer results from the formation of dimers, which in turn associate into a trimer (Fig. 1). The intradimer interface is extensive ( $3200 \AA^{2}, 86$ residues) and tight, stabilized by 19 hydrogen bonds and seven salt bridges. In contrast, the association of three dimers is much weaker, in which the adjacent dimers form an interface measuring $641 \AA^{2}$ involving 20 residues, eight hydrogen bonds and three salt bridges.

The overall fold of an AtNIN2 monomer closely resembles that of Anabaena InvA (PDB entry 5gop; Xie et al., 2016) and InvB (PDB entry 5z74; Xie et al., 2018), which is in line with the high sequence conservation of AtNIN2 with respect to both InvA (56\% sequence identity) and InvB (55\%). Indeed, the superposition of 405 equivalent $\mathrm{C}^{\alpha}$ atoms of the AtNIN2 


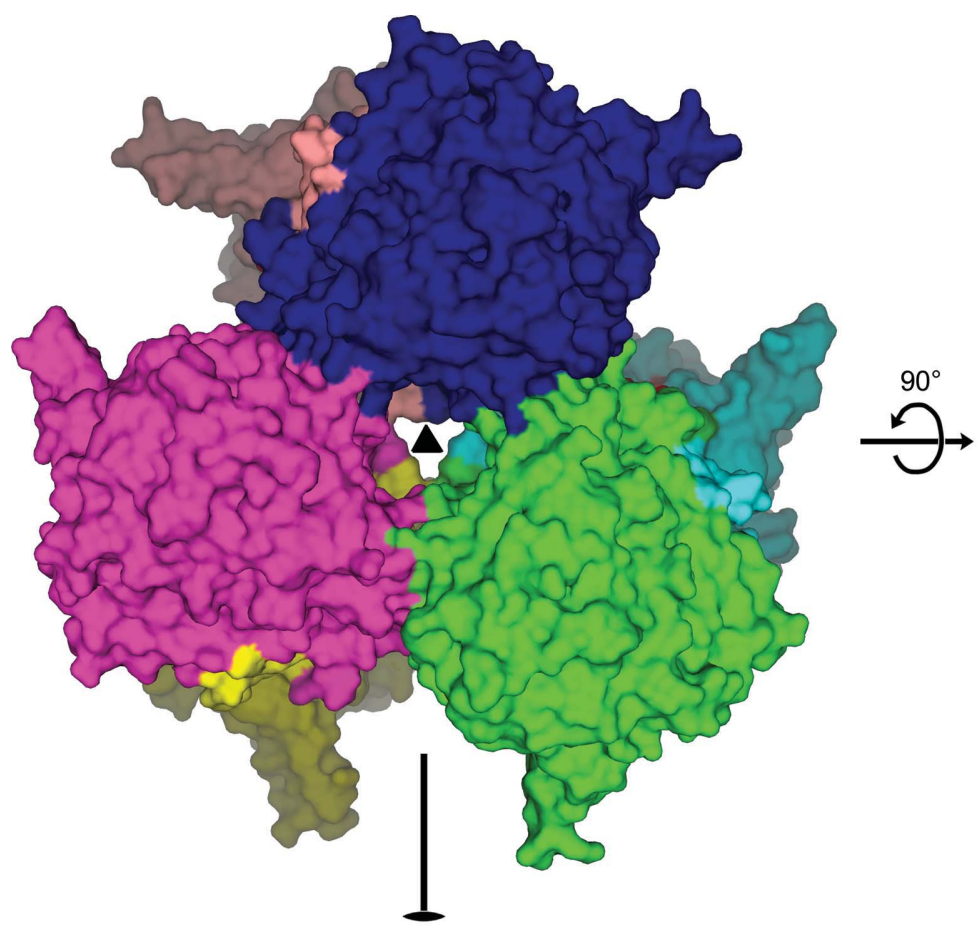

(a)

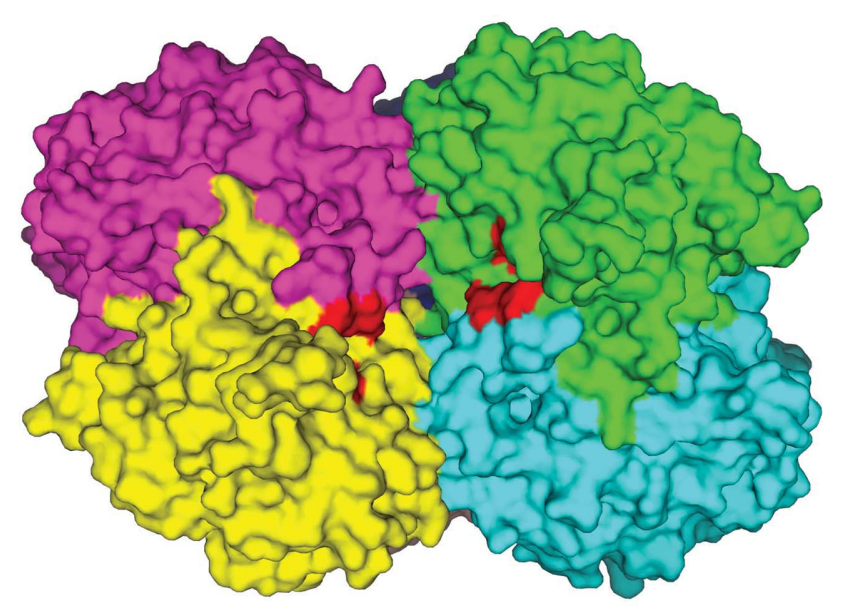

(b)

Figure 1

The AtNIN2 hexamer shown as a surface in two orthogonal views: along the threefold axis $(a)$ and along the twofold axis $(b)$. Symmetry elements are indicated. The residues adjacent to the active site are coloured red.

and InvA monomers yields an r.m.s.d. of $0.6 \AA$ (Fig. 2). It should be noted that InvA has been demonstrated to form stable hexamers in solution (Xie et al., 2016). The hexameric assemblies seen for both AtNIN2 and InvA are highly similar, with an r.m.s.d. of $1.1 \AA$ between 2508 equivalent $\mathrm{C}^{\alpha}$ atoms. At the same time, InvB only forms dimers in solution (Xie et al., 2018), which however closely resemble both the InvA and AtNIN2 dimers seen within the corresponding hexamers. This is in line with the above structural analysis, suggesting a much tighter association of monomers into dimers compared with their subsequent association into trimers.

\subsection{Active site}

The active site of AtNIN2 is formed by Phe124, Arg126, Asp127, Met261, Asp263, Tyr445, His446, Glu489, Gln507 and Trp509, as identified from alignment of the AtNIN2 and InvA structures (Fig. 3). Importantly, all of these residues, as well as the shape of the substrate pocket, are conserved across the AtNIN2, InvA and InvB proteins. Comparison with the InvA and InvB structures in complex with Suc (PDB entries 5gop and 5z74) suggests that AtNIN2 residues Arg126, Asp127, Asp263, Tyr445, His446 and Gln507 should form hydrogen bonds to the substrate, further supported by hydrophobic interactions through the side chains of Phe124 and Trp509 (Fig. 2). Asp263 and Glu489 are expected to be responsible for the catalytic acid-base mechanism, like the corresponding residues of InvA (Asp188 and Glu414). While the surface loop Asn112-Asn119 in close proximity to the active site is disordered in the AtNIN2 structure, the corresponding loop in the InvA structure is involved in substrate binding (Xie et al., 2016).

Hence, the catalytic mechanism of NIs appears to be fully conserved between cyanobacteria and higher plants. Importantly, the close resemblance between the active sites of InvA and AtNIN2 suggests that AtNIN2 should also have substrate stringency towards Suc (Xie et al., 2016), although this needs to be confirmed experimentally. Differences in the $\mathrm{pH}$-activity profile between AtNiN2 and InvA could be linked to the

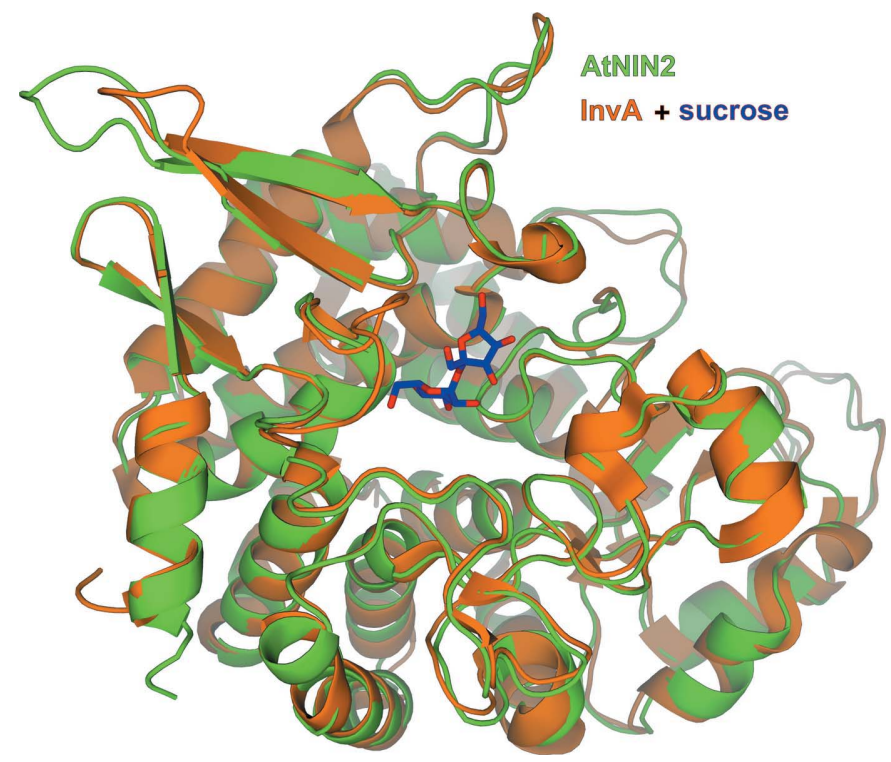

Figure 2

The AtNIN2 monomer (green) superimposed with Anabaena InvA (orange) in complex with sucrose (blue; PDB entry 5gop). 


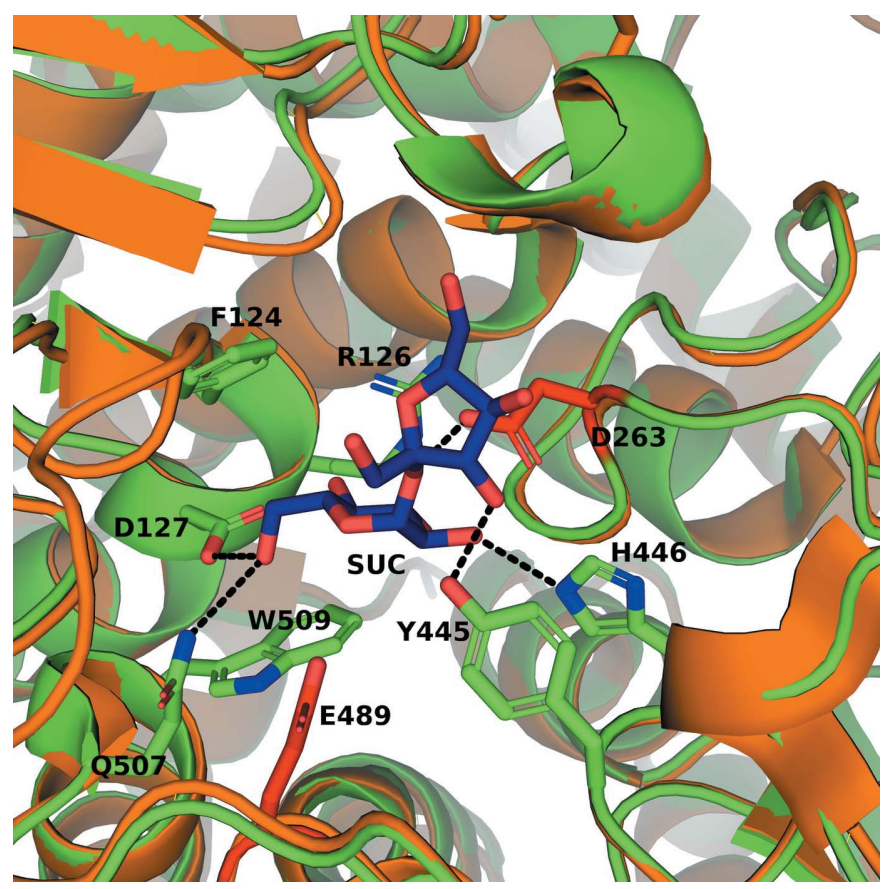

Figure 3

Active sites of AtNIN2 (green) and InvA (orange) compared. Conserved side chains in AtNIN2 are shown as sticks. The nucleophile Asp263 and the acid/base catalyst Glu489 are coloured red. The sucrose molecule bound to InvA is shown in blue ( $\mathrm{C}$ atoms). Hydrogen bonds to the substrate are shown as dashed lines.

differences in the composition of the flexible loop ${ }^{112}$ Asn-ThrThr-Asp-Glu-Val-Asn ${ }^{119}$ in AtNiN2 (disordered in the structure), which is more polar than the corresponding loop ${ }^{40}$ ProGlu-Leu-Ala-Ala-Leu-Asn ${ }^{46}$ in InvA.

\section{Conclusions and outlook}

In this work, we reveal the atomic structure of a member of the GH100 enzymatic family from a higher plant for the first time. The observed sequence and structural similarity between AtNIN2 and InvA/InvB from Anabaena suggests that these enzymes are highly conserved during evolution, at least with regard to their cytosolic isoform. This corroborates the hypothesis that NIs first emerged in cyanobacteria (Vargas \& Salerno, 2010). Our study also confirms the distinct features of NIs from the GH100 family in comparison to acidic invertases from the GH32 family (Xie et al., 2016). While some previous reports have suggested that the NIs of higher plants exist as tetramers or octamers (Chen \& Black, 1992; Liu et al., 2006), our work shows that AtNIN2 forms a hexamer just like InvA. As a next step, it would be interesting to explore the degree to which the structures of mitochondrial or chloroplastic NIs are similar to those of cytosolic NIs, given the distinct differences in $\mathrm{pH}$ within the corresponding subcellular compartments.

\section{Acknowledgements}

The authors are grateful to Tom Struyf (Molecular Plant Biology Laboratory, KU Leuven) for technical assistance and to the ERSF for providing data-collection time.
Funding information

ŁPT was funded by the FWO Vlaanderen, grant G0A4915N.

\section{References}

Alberto, F., Bignon, C., Sulzenbacher, G., Henrissat, B. \& Czjzek, M. (2004). J. Biol. Chem. 279, 18903-18910.

Altenbach, D. \& Ritsema, T. (2007). Recent Advances in Fructooligosaccharides Research, edited by S. Norio, B. Noureddine \& O. Shuichi, pp. 135-156. Trivandrum: Research Signpost.

Barnes, W. J. \& Anderson, C. T. (2018). Plant J. 94, 956-974.

Chen, J. Q. \& Black, C. C. (1992). Arch. Biochem. Biophys. 295, 6169.

Emsley, P., Lohkamp, B., Scott, W. G. \& Cowtan, K. (2010). Acta Cryst. D66, 486-501.

Evans, P. R. \& Murshudov, G. N. (2013). Acta Cryst. D69, 1204 1214.

Gao, J., van Kleeff, P. J. M., Oecking, C., Li, K. W., Erban, A., Kopka, J., Hincha, D. K. \& de Boer, A. H. (2014). Plant J. 80, 785-796.

Kabsch, W. (2010). Acta Cryst. D66, 125-132.

Koch, K. (2004). Curr. Opin. Plant Biol. 7, 235-246.

Liebschner, D., Afonine, P. V., Baker, M. L., Bunkóczi, G., Chen, V. B., Croll, T. I., Hintze, B., Hung, L.-W., Jain, S., McCoy, A. J., Moriarty, N. W., Oeffner, R. D., Poon, B. K., Prisant, M. G., Read, R. J., Richardson, J. S., Richardson, D. C., Sammito, M. D., Sobolev, O. V., Stockwell, D. H., Terwilliger, T. C., Urzhumtsev, A. G., Videau, L. L., Williams, C. J. \& Adams, P. D. (2019). Acta Cryst. D75, 861-877.

Liu, C., Huang, L., Chang, C. \& Sung, H. (2006). Food Chem. 96, 621631.

Maruta, T., Miyazaki, N., Nosaka, R., Tanaka, H., Padilla-Chacon, D., Otori, K., Kimura, A., Tanabe, N., Yoshimura, K., Tamoi, M. \& Shigeoka, S. (2015). New Phytol. 206, 1013-1023.

McCarthy, A. A., Barrett, R., Beteva, A., Caserotto, H., Dobias, F., Felisaz, F., Giraud, T., Guijarro, M., Janocha, R., Khadrouche, A., Lentini, M., Leonard, G. A., Lopez Marrero, M., Malbet-Monaco, S., McSweeney, S., Nurizzo, D., Papp, G., Rossi, C., Sinoir, J., Sorez, C., Surr, J., Svensson, O., Zander, U., Cipriani, F., Theveneau, P. \& Mueller-Dieckmann, C. (2018). J. Synchrotron Rad. 25, 12491260.

Murshudov, G. N., Skubák, P., Lebedev, A. A., Pannu, N. S., Steiner, R. A., Nicholls, R. A., Winn, M. D., Long, F. \& Vagin, A. A. (2011). Acta Cryst. D67, 355-367.

Palmer, W. M., Ru, L., Jin, Y., Patrick, J. W. \& Ruan, Y.-L. (2015). Mol. Plant. 8, 315-328.

Qi, X., Wu, Z., Li, J., Mo, X., Wu, S., Chu, J. \& Wu, P. (2007). Plant Mol. Biol. 64, 575-587.

Roitsch, T., Balibrea, M. E., Hofmann, M., Proels, R. \& Sinha, A. K. (2003). J. Exp. Bot. 54, 513-524.

Ruan, Y.-L. (2014). Annu. Rev. Plant Biol. 65, 33-67.

Shen, L.-B., Yao, Y., He, H., Qin, Y.-L., Liu, Z.-J., Liu, W.-X., Qi, Z.-Q., Yang, L.-J., Cao, Z.-M. \& Yang, Y. (2018). Int. J. Mol. Sci. 19, 224.

Stein, O. \& Granot, D. (2019). Front. Plant Sci. 10, 9.

Sturm, A. (1999). Plant Physiol. 121, 1-8.

Sturm, A. \& Tang, G. (1999). Trends Plant Sci. 4, 401-407.

Tauzin, A. S. \& Giardina, T. (2014). Front. Plant Sci. 5, 293.

Trollope, K. M., van Wyk, N., Kotjomela, M. A. \& Volschenk, H. (2015). FEBS J. 282, 4782-4796.

Vagin, A. \& Teplyakov, A. (2010). Acta Cryst. D66, 22-25.

Van den Ende, W. \& El-Esawe, S. K. (2014). Environ. Exp. Bot. 108, 4-13.

Van den Ende, W., Lammens, W., Van Laere, A., Schroeven, L. \& Le Roy, K. (2009). FEBS J. 276, 5788-5798.

Vargas, W. A. \& Salerno, G. L. (2010). Plant Sci. 178, 1-8.

Weeks, S. D., Drinker, M. \& Loll, P. J. (2007). Protein Expr. Purif. 53, $40-50$. 
Williams, C. J., Headd, J. J., Moriarty, N. W., Prisant, M. G., Videau, L. L., Deis, L. N., Verma, V., Keedy, D. A., Hintze, B. J., Chen, V. B., Jain, S., Lewis, S. M., Arendall, W. B., Snoeyink, J., Adams, P. D., Lovell, S. C., Richardson, J. S. \& Richardson, D. C. (2018). Protein Sci. 27, 293-315.

Winn, M. D., Ballard, C. C., Cowtan, K. D., Dodson, E. J., Emsley, P., Evans, P. R., Keegan, R. M., Krissinel, E. B., Leslie, A. G. W., McCoy, A., McNicholas, S. J., Murshudov, G. N., Pannu, N. S.,
Potterton, E. A., Powell, H. R., Read, R. J., Vagin, A. \& Wilson, K. S. (2011). Acta Cryst. D67, 235-242.

Winter, H. \& Huber, S. C. (2000). Crit. Rev. Plant Sci. 19, 31-67.

Xie, J., Cai, K., Hu, H.-X., Jiang, Y.-L., Yang, F., Hu, P.-F., Cao, D.-D., Li, W.-F., Chen, Y. \& Zhou, C.-Z. (2016). J. Biol. Chem. 291, 2566725677.

Xie, J., Hu, H.-X., Cai, K., Xia, L.-Y., Yang, F., Jiang, Y.-L., Chen, Y. \& Zhou, C.-Z. (2018). FEBS Lett. 592, 1589-1601. 